the absolute production of heat, and then the temperature in the rectum as well as that in the mouth or axilla may be subnormal. As Marey truly points out, when the temperature of the surface is subnormal we must take the temperature in the rectum also before we can decide which of the above causes accounts for the low skin temperature; but I shall have more to say on these points some other time. I must not prolong this paper by a discussion of all the physiological and still less of the pathological bearings of the subject; but if I am right we shall be able (and I have fairly good reason to believe that I am able now, for I have known most of these facts and have been acting on them for a considerable time) not only to control fatigue in physiological conditions but also to prevent its development into such more serious troubles as syncope with dilatation and strain of the heart, or albuminuria, or disintegration of the blood, all of which I take it, and have elsewhere suggested, are but the results of the more serious and prolonged action of uric acid on the circulation, nutrition, and metabolism of the organs and tissues concerned.

Brook-street, w.

\section{THE CLINICAL ESTIMATION OF THE ALKALINITY OF THE BLOOD. ${ }^{1}$}

BY ROBERT HUTCHISON, M.B., C.M. EDIN.,

CHENICAL ASSISTAYT TO THE PROFESSOR OF PHYSIOLOGY, UNIVERSITY OF EDINBURGH.

Ix attempting to estimate the alkalinity of the blood one is confronted by certain initial difficulties. These are dependent partly upon the physical characters of the blood snd partly upon its chemical composition. The albuminous mature of the blood forbids its titration with a mineral acid, and its red colour interferes with the precise detection of the fnal change of colour of the indicator. Further, the blood is not homogeneous, but consists of solid particles, the corwuscles, suspended in a fluid medium, the plasma. In determining the alkalinity of the blood as a whole regard must be had to both these elements. By the separation of the corpuscles it has been found that these contain more alkali than the serum; any method, therefore, which does not rensure thorough breaking down of the corpuscles, with consequent liberation of the alkaline salts therein contained cannot give accurate results. The peculiar chemical composition of the blood gives rise to even greater difficulties. tit must be borne in mind that the blood is not by any means a simple chemical solution, but, on the contrary consists of a highly complex mixture of salts in various and varying combinations. Of these salts, two, the bicarbonate of sodium and the disodic phosphate $\left(\mathrm{Na}_{2} \mathrm{HPO}_{4}\right)$, are chiefly responsible for the alkaline reaction. In both of these salts there is still present an atom of hydrogen replaceable by metal, yet both react alkaline to litmus. Hence the curious paradox which has been emphasised by Drouin, that the blood is chemically an acid fluid, out reacts as alkaline to ordinary reagents. Apart from this, the colour reagent employed to show when the neutral point is reached has a considerable influence upon the result obtained. Thus we find that solutions of monobasic salts of phosphoric acid-e.g., $\mathrm{NaH}_{2} \mathrm{PO}_{4}$ -are acid to litmus, but neutral to phenol-phthaleine; dibasic salts of the same acid-e.g., $\mathrm{Na}_{2} \mathrm{HPO}_{4}$-are alkaline to litmus, but, unless in strong solution, also neutral to phthaleine. Now, as such salts are always present in the blood the result we obtain will obviously depend upon whether we employ litmus or phthaleine as our indicator. In addition to this $\mathrm{CO}_{2}$ is always present in the blood, and when liberated by another acid escapes and affects litmus feebly or not at all. In the case of phosphoric acid compounds, on the other hand, the acid liberated is able to act upon litrous as acid. Hence a blood, the alkalinity of which is chiefly due to phosphate of soda, will give an apparently lower alkalinity than one which contains a larger proportion of sodium bicarbonate. Nor does this exhaust the ist of difficulties. It has been specially insisted upon by Mlever ${ }^{2}$ that we have no right arbitrarily to select a certain

1 This article contains the substance of an essay which was awarded the Edinburgh University London Club Prize in August, 1895. - Meyer: Archiv für Experimentale Pathologie und Pharmacie, Band xivi., 1883. reagent-e.g., litmus-and exclude from the category of acids all substances which fail to give a red reaction with it. Thus some alcohols, some amido acids, and some amides are chemically acids but do not react as such to litmus, and these may exist in the blood. Some of the higher fatty acids, also, when liberated are insoluble in water and so cannot affect the litmus.

When these difficulties are considered one might at first be tempted to abandon as futile any attempt to gauge clinically the alkalinity of the blood. The pbysical difficulties can, however, be overcome by suitable methods, while as regards the chemical objections it must be confessed that many of them are rather theoretical than practical. It is true that the blood contains chiefly acid salts ; it is also true that it contains real acids. The balance of the reaction, however, is always in favour of what is commonly understood as alkalinity. Our methods should be able to show us to what extent fluctuations in this alkalinity occur, although we cannot expect them to inform us as to what substance it is which, by its presence or absence, occasions the fluctuations. Those substances which, while chemically acids, do not react as such to litmus are not likely to be present in the blood in such amounts as materially to affect our results. After these preliminary observations we may turn now to the consideration of the methods which are at our disposal for estimating the alkalinity of the blood. I shall confine myself in this article to methods of clinical applicability. Obviously we cannot justly demand from such a method very great accuracy. Like all clinical methods, it must needs be more or less of a compromise between accuracy and simplicity. Zuntz ${ }^{3}$ was one of the first to attempt the estimation of the alkalinity of the blood. He employed titration with phosphoric acid, but his method demanded too much blood to be of use clinically. The same criticism applies to the method of Lassar ${ }^{*}$ who employed tartaric acid instead of phosphoric. Liebreich ${ }^{5}$ devised a method for showing the alkaline reaction of the blond by means of plates of plaster-of-Paris impregnated with litmus. When a drop of blood was placed upon the plate the plasma soaked in and affected the litmus while the corpuscles remained on the surface and could be wiped off without obscuring the result. Renzi ${ }^{6}$ employed this method to estimate variations in the alkalinity, but as he was guided solely by the intensity of the blue colour produced his results were not accurate. Lépine ${ }^{7}$ and Canard ${ }^{8}$ were the first to make extensive observations in man by the titration method, but employing comparatively large quantities of blood. It was not till a decade ago that Landois ${ }^{9}$ introduced a titration method which required only easily obtainable quantities of blood. As this is the type of the titration method now in use it demands a word of description. The principle of the method consists in preventing coagulation of the blood by means of a saline solution, while all the alkali present is neutralised by titration with tartaric acid, litmus paper being employed to show when the neutral point is reached. Sulphate of soda solution is prepared, and to this a $\frac{7 \cdot 5}{1000}$ solution of tartaric acid is added in graduated amounts.

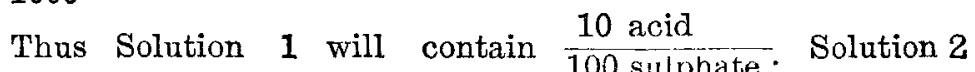

20 acid

$\overline{90 \text { sulphate; }}$ and so on up to a solution which contains 100 acid

10 sulphate.

Equal quantities of one of these solutions and of the blood to be examined are sucked up into a fine pipette, mixed in a watch-glass and the reaction tested with sensitive litmus paper. If the solution is still alkaline the next strongest acid solution is taken, and so on till the result is neutral. This method has been largely employed by von Jaksch, ${ }^{10}$ who drew the blood with cupping glasses, and by Peiper, ${ }^{11}$ who found the latter unnecessary. The method has found its most recent advocate in Drouin, ${ }^{12}$ whose thesis constitutes the most comprehensive monograph on the

3 Zuntz : Centralblatt für die medicinischen Wissenschaften, 1867.

4 Lassar: Archiv für die gesammte Physiologie, Band lxviii.

5 Liebreich: Berichte der deutschen chemischen Gesellschaft, Band i., p. $48,1868$.

$$
\begin{gathered}
\text { 6 Renzi : Virchow's Archiv, Band cii., } 1888 . \\
7 \text { Lépine: Gazette Médicale, 1879. } \\
8 \text { Canard: Thèse, Paris, 1878. }
\end{gathered}
$$$$
9 \text { Landois : Réal. Fncyclopédie, iii. } 1884
$$

10 Von Jakseh : Zeitschrift für klinische Medicin, Band xiii., 1888 11 Peiper: Virchow's Archiv, Bavd cxvi., 1889

12 Drouin: Hémo-alcalimétrie et Hémo-acidimétrie, Thèse, Paris, 1892 
alkalinity of the blood which has yet appeared. He has improved slightly upon the method of Landois by using a $\frac{n}{30}$ oxalic acid solution to mix with the sulphate of soda. With a special dropper he places in the first glass one drop of acid and nine of sulphate, the second receives two of acid and eight of sulphate, and so on up to the tenth. An equal amount of blood is then added to each glass and rapidly titrated, delicate litmus paper being used to indicate in which glass the reaction is neutral. The method is, on the whole, simpler than that of Landois and quite as accurate. Now all these methods have the drawback of being somewhat elaborate and of demanding for their proper application a considerable amount of blood, of apparatus, of practice, and of time. It was, therefore, much to be desired that a simpler method might be found. Such a method Haycraft and Williamson believed to have discovered and described it in the proceedings of the Royal Society of Edinburgh for 1888. Since then their method has been recommended for use by various works dealing with clinical methods. ${ }^{13}$ It depends upon the fact that if a drop of blood be placed upon the surface of a neutral glazed litmus paper the glaze prevents the corpuscles from entering the interstices of the paper while the plasma is able to soak in, and on wiping away the drop the blue colour due to the alkalinity of the plasma is distinctly seen. The fact that the alkalinity of the blood could be demonstrated in this manner was pointed out some years ago by Zuntz and by Schäfer. It occurred to Haycraft and Williamson that by treating litmus paper with different strengths of acid and then glazing them one would get a series of papers of graduated acidity, one of which would just be neutralised by the alkalinity of normal blood. All the papers more strongly acid than this would remain unaltered on wiping away the drop of blood, while all the less acid papers would exhibit a blue reaction, which would be the more strongly marked the less acid the paper.

This method seems at first sight to be a great advance upon the titration procedures. It has the undoubted merit of simplicity and can be very rapidly carried out. Haycraft stated that by its means " he had found the reaction of the blood to vary as greatly as that of the urine." Hence it would seem to answer every requirement. I prepared a series of ten papers according to the instructions of the authors of the method. Sulphuric acid was used to acidify the papers. The strongest paper (No. 1) was soaked in a normal solution of the acid. No.2 was soaked in a half normal solution, and so on up to 10 , which was soaked in a decinormal solution. Having determined which paper just reacted to normal blood I made a series of observations on the alterations in the alkalinity in disease by means of the papers. Of course, the results afforded could only be approximate, as there is a considerable difference between, say, a half normal and a onethird normal solution of sulphurie acid, but in this respect the method would not compare unfavonrably with the titration method of Landois. Nor did I find that there was much force in the objection-the only one I have hitherto seen advanced against the method-that there is difficulty in determining exactly which is the paper which just gives a blue reaction with the drop of blood. It was only later, on coming to compare my results with those of other workers obtained by the titration method, that it was found that while in many diseases the results coincided, in others marked discrepancies occurred. This was specially noticeable in the case of the anæmias. I invariably found that the alkalinity of the blood in anæmia as tested by the method of Haycraft and Williamson was above normal, and often apparently to an excessive degree. Now if there be one fact upon which all those who have employed the titration method are agreed it is this-that in anæmia the alkalinity of the blood is reduced. How, then, is this contradiction in the results afforded by the two methods to be explained? It might at first be asserted that the results of the two methods are not strictly comparable. It is obvious that by the glazed litmus paper method the alkalinity of the plasma is what is ascertained. The corpuscles being left on the surface of the paper the alkali contained in them does not get the chance of affecting the litmus. By the titration method, on the other hand, the corpuscles are more or less completely broken down and the alkali which they contain is liberated and so arrives at estimation. But this explanation

13 See von Jaksch's Clinical Diagnosis, third edition, 1894 ; Finlayron's Clinical Manual, \&c will not suffice, for the difficulty was to discorer why a method which takes no account of the corpuscles should yet give an apparently higher alkalinity than one which estimates the alkali of all the components of the blood together. In searching for another explanation it occurred to me that the more watery condition of the blood in most anæmias might be the cause of the different result yielded by the two methods. The more watery the blood is the more readily will it percolate into the glazed litmus paper and the more readily will the acid be neutralised. That this supposition was correct was easily proved by experiment. If one takes fifty c.c. of glycerine and mixes thoroughly with it one c.c. of a normal solution of caustic soda the mixture will be found to react to about paper 3 of our series. If now one adds to the mixture twenty c.e. of water and again mixes the solution will now be found to react to a considerably more acid paper. That is to say, the solution, although really more dilute than before, is apparently more strangly alkaline-a paradox which is only to be explained by the greater ease with which the more watery fluid percolates into the paper. This diffculty cannot be got over by allowing the drop of blood to remain all the longer on the paper because, as will be seen, the alkalinity of the blood diminishes rapidly after its withdrawal from the body. Seeing, then, that the results afforded by the glazed litmus paper method are 80 dependent upon the consistency of the blood (a by no means constant factor) $I$ consider that it is not a trustworthy clinical method and must be discarded. We are, therefore, driven back upon the use of the titration method. We have seen that this method tells us the total amount of alkali in the blood, not the alkalinity of the plasma only. But it will only give us this result if properly carriec out. Loewy ${ }^{14}$ has shown that unless the blood be titrated slowly and at the body temperature all the corpuscles are not broken down and the total alkalinity is therefore not detected, and he contends that, owing to neglect of this precaution, previous results have given too low an estimate.

I turn now to a consideration of the practical results which the estimation of the alkalinity of the blood bas afforded. Before drawing any conclusions as to the alterations of the alkalinity due to disease we must, of course, make ourselves acquainted with the variations which occur under physiological conditions. The first point to emphasise regarding the alkalinity of healthy blood is its great constancy. This constancy is the more remarkable when one reflects upon the varying amount of alkali which must be entering the blood stream at different times. The only circumstances in which it is appreciably altered are during the ingestion of food and after severe muscular work. The alkalinity is considerably increased in the former condition and decreased as the result of the latter. The increase after meals is synchronous with the appearanee of the "alkaline tide" in the urine, and the latter is to be regarded as the expression and result of the former. The increased alkalinity is probably to be explained, in part at least, by the absorption of alkaline salts from the food. In part it may be due to the discharge into the circulation of sodium carbonate or bicarbonate resulting from the production of hydrochloric acid from sodium chloride in the secreting cells of the stomach. The effect of severe muscular work is to cause a fall in the alkalinity of the blood, this being probably due to the production of considerable quantities of lactic acid by muscular metabolism. Turning attention now to the condition of the blood in disease we find that the constancy above referred to is maintained in a very remarkable manner. There are comparatively few diseases in which the alkalinity of the blood is appreciably altered. By what mechanism the balance of alkalinity is maintained we know not, but this we know-that it is a remarkably perfect mechanism. Whenever the amount of alkali in the blood rises much above the mean. the excess appears to be promptly excreted by the kidney. In the reverse direction, however, the mechanism does not seem to act so well. Considerable quantities of acid products can accumulate in the blood without being got rid of. Hence reduction of alkalinity is very much more frequently found in disease than increase. One may say, indeed, that an increased alkalinity of the blood is, with perhaps one exception, never found as the result of disease. Now, there are many morbid conditions in which one would not expect $a$ priori to find any alteration of the alkalinity of the blood, and this expectation is usually found to be justified. There

14 Loewy : Archiv fuir die gesammte Physiologie, Band lviii., 1894. 
are others in which, from what we already know of their pathology, some alteration might naturally be looked for. The latter may usually be referred to one of four groups: (1) all diseases associated with profound alterations of the general metabolism-e.g., fevers and cachexias ; (2) diseases of the blood itself; (3) some toxic states-e.g., jaundice, uræmia, and advanced diabetes; and (4) some so-called diathetic conditions-e.g., gout and rheumatism. Now, as a matter of fact, as far as our observations have hitherto gone, the above list includes practically all the diseases in which any marked alteration in the alkalinity of the blood has been vound to occur. I shall consider briefly each group by itself.

1. Speaking generally, one may say of fever as a process that it is accompanied by a diminution of the alkalinity of the blood. This diminution, though very constant, is not invariable, and is found to be roughly proportionate to the height of the fever but not to its duration. It is most probably occasioned by the entrance into the circulation of imperfectly oxidised katabolic products of an acid nature, which are laid hold of by the alkaline salts in the blood and so neutralised. We do not know that any of the general symptoms of fever can be referred to this reduced alkalinity or that it would be at all advisable to make it the basis of any special line of treatment. Yet the reduced alkalinity of the blood is undoubtedly, per se, unfavourable to tissue vitality. It has been shown by Castellino and Cavazzani 15 that alkaline Iiquids stimulate the protoplasmic movements of leucocytes while contact with liquids poor in alkali seems to render them torpid. It is interesting to collate with this the further fact observed by Fodor ${ }^{16}$ that an increased alkalinity of the blood renders animals much more resistant to organisms. The bearing of these observations upon the pathology of ferer is obvious. In cachexia, however induced, the blood is also less alkaline than normal, but this may be due in part, at least, to the poverty of blood with which this condition is associated.

2. I have stated in an earlier part of this article that in almost all the anæmias the alkalinity of the blood is reduced. This is true of simple anæmia, of pernicious anæmia, and of leucocythrmia. The causes of the reduction are probably several. Seeing that the red cells contain a considerable quantity of alkali any reduction in their number must of itself entail a corresponding reduction in the total alkalinity of the blood. This explanation is supported by the fact that in cases of pure chlorosis-i.e., where the hæmoglobin is alone reduced-the alkalinity of the blood is not diminished, may, indeed, be increased. Deficient oxidation of the tissues no doubt plays a part just as it does in fever. Uric acid has been found in the blood in anæmia by ron Jaksch ${ }^{17}$ and Spiro ${ }^{1 *}$ has demonstrated the presence of lactic acid in encocythæmia. It is also possible that in anæmia the mineral constituents of the blood are reduced just as the cellular are, the blood being really more watery than normal. Here, again, I would point out that the diminished alkalinity of the blood cannot be made a reason for the administration of alkalies in anæmia. Iron and arsenic are both drugs which tend to reduce rather than to increase the alkalinity of the blood, yet the utility of both in anzemia is andoubted and the mineral acids are probably more helpful than alkalies.

3. In all the toxic conditions mentioned above the alkalinity is also diminished. In jaundice this is to be attributed to the presence of bile acids in the blood. In uramia the ceduction is often very marked, and this is the more noteworthy as renal disease does not by itself lead to any diminution of alkalinity. The fact is an additional argucont, were one needed, against the ammonia theory of aræmia. In diabetes the alkalinity tends to be always low, but is especially reduced if coma supervenes. Its occurrence is accounted for by the entrance into the circulation of oxybutyric acid.

4. I cume finally to certain "diathetic" conditions in which the investigation of the alkalinity of the blood is of peculiar interest. I refer to gout and rheumatism. In the former the allinalinity of the blood is stated to be lower than in any other disease, but this is just what we would expect from the excess of uric acid in the circulation. "The old "acid" theory of acute rheumatism lends to the determination of the alkalinity of the blood in that disease a

I5 Castellino anil Cavazzani : Gazetta degli Ospitale, 1833.

Todor: Centralblatt tur Bakteriologie und Parasitent uinde, 1895.

17 Von Jaksch: Ueber Uric Acidamie. Deutsehe Medicinische Woichenschrift, 1390

is Spiro: Zeitsehrift fur Physiologische Chemie, Band i. special importance. Is the alkalinity reduced? 'The seply is definitely and almost unanimously in the negative. Pfeifer, von Jacksch, and Drouin have all failed to find any reduction, and with their observations those of the present writer agree. This is confirmatory of the results of special analyses, Garrod ${ }^{19}$ having failed to find any uric acid in the blood in acute rheumatism, while Salomon ${ }^{20}$ has proved the absence of lactic acid. In chronic articular rheumatism and in rheumatic arthritis the alkalinity would appear to be sometimes reduced, but this may be due to accompanying anæmia.

I may refer lastly to the effects of drugs on the reaction of the blood. These bring out very clearly the existence of the regulating mechanism. It has been found almost impossible to affect the reaction of the blood for any length of time by the administration of acids or alkalies by the mouth. Hoffmann fed pigeons on substances yielding only an acid ash, but found that alkalies were retained in the blood to form salts with the acids. This occurs even when more acid is administered than would suffice to neutralise all the alkali in the body. Lassar ${ }^{21}$ got only slight effects from the administration of even large quantities of alkalies and acids, and his results have been confirmed by those of other observers. A few of the so-called "alteratives," such as arsenic and phosphorus, have been found to produce some diminution of alkalinity, probably by lessening tissue oxidation. In carbonic oxide poisoning a considerable reduction occurs for the same reason; in this case the presence of considerable quantities of lactic acid in the blood has been demonstrated (Araki). Our comparative inability appreciably to alter the reaction of the blood by means of drugs is rather remarkable, although therapeutically somewhat disappointing.

I may now, in closing, briefly summarise the contents of this article. We have seen that the clinical determination of the alkalinity of the blood is rendered difficult alike by physical and by chemical considerations, tnat the existing methods have been devised with the object of surmounting these difficulties, and that these methods are for practical purposes only two in number-the titration method (Landois) and the glazed litmus paper method Haycraft and Williamson). I believe I have been able to show that the results furnished by the second of these methods are unreliable, inasmuch as the reaction is determined largely by the viscidity of the blood examined, a factor of great inconstancy. The titration method is, therefore, alone available. The results of this method apply to the alkalinity of the blood as a whole, but only when titration is carried out slowly and at the body temperature. We are thus left in ignorance as to how much of the alkali in any given case is contained in the blood-cells and how much in the plasma. We have seen, further, that the clinical application of this method has brought into prominence one striking fact regarding the alkalinity of the blood-its constancy. That this constancy is maintained both in health and disease and is apparently the result of a regulating mechanism. The alterations in disease are practically always in the direction of diminution of alkalinity, induced probably by the entrance of acid substances into the circulation. Thus, lactic acid is present in leucocythæmia, carbonic oxide poisoning, \&c., uric acid in gout and in anæmia, oxybutyric acid in diabetes, and bile acids in jaundice. In addition to these conditions we saw that the alkalinity is reduced in ursemia, in all febrile states, and in anremias with the exception of chlorosis. The reasons of the reduction in these different diseases I have briefly discussed. Lastly, we have seen that, owing to the existence of the regulating mechanism, we are practically unable materially to influence the reaction of the blood by the administration of drugs. As the number of available observations by different workers is now fairly large, and covers nearly every known disease, we are entitled to conclude that not much more will be made out by existing metbods, and on reviewing the practical results of the information acquired one is bound to confess that they are rather meagre. These results certainly throw side lights, even if of a negative character, on some pathological questions, but as regards direct clinical bearing it is, I think, evident that the information to be derived from the estimation of the alkalinity of the blood in any given case is hardly commensurate with the expenditure of time and trouble which such estimation entails. Eainburgir.

Garrod: Heynolds's Srstem, first edition. 20 Salomon: Jahrest ericht für 'Iherchenie. Hand $x$. 1. Lassiri : Aruip tur die gezamme l'iy siologie, Band ixxiv. 